\title{
Common variable immunodeficiency: different faces of the same disease
}

\author{
Elżbieta Grześk, Anna Dąbrowska, Anna Urbańczyk, Marlena Ewertowska, Mariusz Wysocki, Sylwia Kottan
}

Department of Paediatrics, Haematology and Oncology, Collegium Medicum in Bydgoszcz, Nicolaus Copernicus University in Torun, Poland

Adv Dermatol Allergol 2021; XXXVIII (5): 873-880

DOI: https://doi.org/10.5114/ada.2021.110067

\begin{abstract}
Introduction: Common variable immunodeficiency (CVID) is one of the primary humoral immunodeficiencies. Despite the inborn nature, the first symptoms may appear in both children and adults. It is characterized by hypogammaglobulinaemia, severe infections, autoimmunity, allergies, and a predisposition to cancer. A delay in diagnosis is a significant problem: the time from the first symptoms of the disease to diagnosis and the implementation of proper treatment is usually very long. The consequence can be irreversible complications, which is why it is so important to promote knowledge on this immunodeficiency.

Aim: To present the clinical and laboratory manifestation of primary immunodeficiencies such as common variable immunodeficiency.

Material and methods: The study presents the clinical and laboratory phenotype of 14 patients diagnosed with CVID, aged 5 to 58 years. A detailed medical history was taken, and clinical symptoms, immunological test results and complications were analysed in each patient. According to the ESID guidelines, in the differential diagnosis process of CVID the secondary hypogammaglobulinaemia was excluded.

Results: The follow-up period ranged from 39 to 133 months (median: 79 months). The median delay for the entire group was 5 years, which was shorter in children than in adults. In the presented group, the infectious phenotype (pneumonia, sinusitis) was dominant. Autoimmune and allergic diseases, malignant tumours and enteropathies have also been observed.

Conclusions: The diagnostic delay is still too long, especially in adults, which can lead to serious and irreversible complications. Early diagnosis and appropriate treatment with intravenous and subcutaneous immunoglobulins reduces the frequency of infections and their potential complications.
\end{abstract}

Key words: immunodeficiency, clinical course, diagnosis, treatment, immunoglobulins.

\section{Introduction}

Variable common immunodeficiency (CVID) is a primary, humoral immunodeficiency with a very diverse clinical picture. As knowledge about primary immunodeficiency (PID) advances, individual diseases, including CVID, are diagnosed more effectively. Currently, the incidence is estimated at $1: 25,000[1-3]$.

According to the definition, diagnosis of CVID is possible $>4$ years of age, although its clinical symptoms may appear earlier. The first peak of incidence is observed in the $1^{\text {st }}$ decade of life, the second - in the $2^{\text {nd }}$ and $3^{\text {rd }}$ decades of life $[4,5]$.

The incidence of CVID is influenced by environmental, epigenetic and genetic factors.
So far, this deficiency has been associated with mutations in 13 genes, and the most common mutation concerned the TNFRSF13B (TACI) gene [3, 6-8]. Direct sequencing of genes associated with CVID allows the determination of the genetic basis in a small percentage of patients (3-10\%). The increasing use of the Next Generation Sequencing (NGS) tests in patients with CVID causes the molecular basis to be found much more often [8]. The following monogenic disease entities are distinguished, e.g., activated PI3K delta syndrome (APDS) or autoimmune lymphoproliferative syndrome (ALPS), which have a phenotype similar to CVID, but require a different therapeutic approach, e.g. specific biological therapy or stem cell transplantation [1, 5, 8-12].

Address for correspondence: Elżbieta Grześk MD, PhD, Department of Paediatrics, Haematology and Oncology, Collegium Medicum, Nicolaus Copernicus University, 9 Skłodowskiej-Curie St, 85-094 Bydgoszcz, Poland, phone: +48 52 585 4860, fax: +48 5854867, e-mail: ellag@cm.umk.pl Received: 24.02.2021, accepted: 6.08.2021. 
The clinical picture of CVID includes an infectious phenotype with a rich course and various aetiology of infections, an autoimmune and allergic phenotype, as well as a strong tendency to non-malignant lymphoproliferation and the occurrence of neoplastic diseases, especially lymphomas and gastric cancer [3, 5, 6, 12-15]. Most often, patients display mixed phenotypes, with a varied combination of symptoms and comorbidities. This hinders and delays the correct diagnosis. The lack of diagnosis results in the lack of appropriate treatment, which often translates into irreversible infectious and non-infectious complications [2, 12-14, 16-18].

In addition to promoting knowledge on CVID, it is important to use appropriate diagnostic criteria to diagnose this immunodeficiency. At present, physicians follow the diagnostic criteria developed by the ESID (European Society for Immunodeficiencies) [4, 5, 12, 19, 20]. The last update of the ESID criteria took place in 2016 [20]. They are presented in Table 1.

\section{Aim}

Due to the fact that CVID is a great challenge for physicians, we present our own experience in terms of diagnosis and treatment of these patients.

\section{Material and methods}

The study included 14 patients with a clinical and laboratory CVID phenotype, aged 5 to 58 years. The study group was dominated by children (10 patients, 71.4\%). Before 2016, 4 (28.6\%) adults were diagnosed at the Immunology Clinic for Children, when there was no clinic for adults in the Kujawsko-Pomorskie Voivodeship. Based on the ESID criteria [20], CVID was diagnosed in patients after 2016, and the previous diagnosis was verified. According to the ESID guidelines, in the differential diagnosis process of CVID, the secondary hypogammaglobulinemia was excluded. A detailed medical history was taken and a physical examination was performed in each patient. Particular attention was paid to the pres- ence of symptoms and diseases accompanying PID as well as their complications. The time from the onset of the first symptoms of CVID to the time of correct diagnosis (the diagnostic delay) was analysed. Each patient had a defined CVID clinical phenotype.

Each patient underwent a panel of immunological tests necessary for diagnosis in accordance with the ESID criteria (immunoglobulin concentration in the main classes and IgG1-4 subclasses, post-vaccination response, the presence of isohemagglutinins as natural antibodies, distribution of lymphocyte subpopulation). Selected patients underwent NGS tests. Tests were also performed to assess the efficiency of individual organs, depending on the symptoms observed in individual patients.

The method of treating patients was analysed, taking into account the administration of human normal immunoglobulins by the intravenous and subcutaneous routes. The clinical response to the treatment (reduction of infections) was assessed and the IgG level was monitored during the treatment.

\section{Ethics approval}

This study was performed in line with the principles of the Declaration of Helsinki. Approval was granted by the Ethics Committee of Nicolaus Copernicus University in Torun, Collegium Medicum in Bydgoszcz (No. 161/2014).

\section{Results}

CVID was diagnosed in 14 patients, including 6 (42.85\%) females and 8 (57.14\%) males. Table 2 presents the delay in diagnosis for each patient. It was between the age of 1.5 to 18 . The median delay for the entire group was 5 years. When analysing children and adults separately, the delay was between the age of 1.5 and 7 in the paediatric population, and between 5 to 18 years of age in adults (Table 2).

The infectious phenotype was dominant among the subjects, both in children and adults (Tables 3 and 4).

Table 1. CVID criteria according to the ESID guidelines. So far, CVID has been diagnosed as probable or possible according to the ESID criteria [20]. In 2016, these criteria were updated. At the moment CVID can be diagnosed in the following situations

I The occurrence of at least one of the following: increased susceptibility to infection, autoimmune manifestations, granulomatous disease, unexplained polyclonal lymphoproliferation, positive family history

II And decreased IgG and IgA levels, with or without the decrease of IgM level (measured at least twice, less than 2 standard deviations of the normal levels for the age)

III And at least one of the following: abnormal, poor response to vaccines and/or absence of isohemagglutinins, absence of the protective level of specific antibodies despite vaccination, low switched memory B-cells (less than $70 \%$ of the age-related normal value)

IV And exclusion of secondary causes of hypogammaglobulinaemia

$\checkmark$ And a diagnosis after the age of four, although clinical symptoms may occur earlier

VI And no evidence of profound T-cell deficiency 
Table 2. Diagnostic delay and mean follow-up in individual patients with CVID

\begin{tabular}{|c|c|c|c|c|}
\hline Patient & Age - first symptoms [years] & Age - first diagnosis [years] & Diagnostic delay [years] & $\begin{array}{l}\text { Mean follow-up in DPHO* } \\
\text { [months] }\end{array}$ \\
\hline 1 & 10 & 16 & 6 & 104 \\
\hline 2 & 18 & 26 & 8 & 45 \\
\hline 3 & 12 & 17 & 5 & 133 \\
\hline 4 & 19 & 27 & 8 & 41 \\
\hline 5 & 40 & 58 & $18 ! ! !$ & 39 \\
\hline 6 & 30 & 35 & 5 & 61 \\
\hline 7 & 7 & 13 & 6 & 92 \\
\hline 8 & 9 & 12 & 3 & 52 \\
\hline 9 & 2 & 5 & 3 & 125 \\
\hline 10 & 3.5 & 5 & 1.5 & 109 \\
\hline 11 & 10 & 13 & 3 & 97 \\
\hline 12 & 6 & 13 & 7 & 108 \\
\hline 13 & 11.5 & 16.5 & 5 & 66 \\
\hline 14 & 8 & 10 & 2 & 44 \\
\hline Median & 10 & 14.5 & 5 & 79 \\
\hline Min. & 2 & 5 & 1.5 & 39 \\
\hline Max. & 40 & 58 & 18 & 133 \\
\hline
\end{tabular}

*The Department of Paediatrics, Haematology and Oncology in Bydgoszcz.

Pneumonia was the most common (12/14 patients; $85.7 \%)$. In 3 children, pneumonia was complicated: respiratory failure occurred in 1 (7.14\%) patient, pleural empyema was observed in 3 (21.4\%) patients and granulomatous and lymphocytic interstitial lung disease (GLILD) in $2(14.3 \%)$ cases.

Sinusitis was frequent in the clinical picture, and its spectacular course, with the necessity to perform functional endoscopic sinus surgery (FESS), occurred in $9(64.3 \%)$ patients. In one of the patients, an infection with EBV and CMV (in the form of lymphadenopathy, hepatosplenomegaly, leucocytosis with lymphocytosis, biochemical features of hepatitis) the disclosure of immunological disorders preceded by 3 years allowing for the diagnosis of CVID.

Features of impaired immune regulation with autoimmune manifestations and/or allergy in various forms (scleroderma, Raynaud's disease, immune thrombocytopenia, autoimmune haemolytic anaemia, complete alopecia, Crohn-like diseases, skin pemphigus, chronic urticaria, atopic dermatitis) were observed in 6 patients, 42.9\% (Tables 3 and 4). One patient, in whom CVID was diagnosed 18 years after the first symptoms suggestive of PID, was diagnosed with lung cancer. In this case smoking was an additional factor contributing to the development of this cancer.
In the presented group of patients with one person (a 13-year-old girl) diagnosed with CVID, the typical CVID picture was associated with a short stature and microphthalmia. Noonan syndrome was suspected but was not eventually confirmed. Unfortunately, no NGS testing was performed, which would increase the chance to diagnose the molecular basis of the probable syndrome associated with PID.

Table 5 presents the immunoglobulin levels in main classes and subclasses at the moment of diagnosis and during intravenous administration of human immunoglobulins. Table 6 presents the results of the primary lymphocyte subpopulations at the moment of diagnosis. The presented results obtained from patients at diagnosis were the basis for the diagnosis of CVID, in accordance with the ESID criteria. On the other hand, the measured IgG levels during the replacement therapy allowed to monitor its effectiveness (Table 5). One patient had an extremely low percentage of $B$ lymphocytes, which is typically not seen in CVID. Unfortunately, while the patient was under paediatric care, we were unable to perform genetic testing for $\mathrm{X}$-linked agammaglobulinemia (XLA) and verify the diagnosis.

The follow-up time from diagnosis was between 39 and 133 months (median 79 months). All patients underwent human immunoglobulin replacement therapy and, 
Table 3. Clinical characteristics of individual patients

\begin{tabular}{|c|c|c|c|}
\hline Patient & Sex & $\begin{array}{c}\text { Age at } \\
\text { diagnosis }\end{array}$ & The most common symptoms \\
\hline 1 & $\mathrm{~F}$ & 16 & $\begin{array}{l}\text { Sinusitis, pharyngitis, urinary tract infections, oral candidiasis, erythrocyturia, chronic urticaria, } \\
\text { lymphadenitis, skin warts }\end{array}$ \\
\hline 2 & $\mathrm{~F}$ & 26 & Pneumonia, sinusitis, pharyngitis \\
\hline 3 & M & 17 & $\begin{array}{l}\text { Pneumonia, sinusitis, cellulitis, pharyngitis, urinary tract diseases, skin abscesses, bronchiectasis, } \\
\text { splenomegaly, lymphadenopathy, anaemia, leucocytosis, hand swelling, systemic sclerosis, Raynaud's } \\
\text { syndrome, acne, erythrocyturia, nephrolithiasis }\end{array}$ \\
\hline 4 & M & 27 & Recurrent pneumonia, bronchitis, sinusitis \\
\hline 5 & M & 58 & $\begin{array}{l}\text { Pneumonia, bronchitis, bronchiectasis, urinary tract disease, nephrectomy, pulmonary segmentectomy } \\
\text { caused by infectious complications, squamous cell carcinoma of the lung }\end{array}$ \\
\hline 6 & $\mathrm{~F}$ & 35 & Sinusitis, pharyngitis, lymphocytic pneumonia, arthritis, neutropenia, thrombocytopenia, anaemia \\
\hline 7 & M & 13 & Sinusitis, lymphadenitis, atopic dermatitis, hepatomegaly, drug-induced alopecia, gynecomastia, epilepsy \\
\hline 8 & M & 12 & $\begin{array}{l}\text { Pneumonia, sinusitis, otitis media, recurrent pharyngitis, lymphadenitis, sepsis, GLILD, chronic diarrhoea, } \\
\text { atopic dermatitis }\end{array}$ \\
\hline 9 & M & 5 & $\begin{array}{l}\text { Pneumonia, pleural empyema, cytomegalovirus and Epstein-Barr virus infection, chronic diarrhoea, } \\
\text { gastritis, colitis, hepatosplenomegaly }\end{array}$ \\
\hline 10 & M & 5 & $\begin{array}{l}\text { Pneumonia, sinusitis, chronic bronchitis, gingivitis, chronic diarrhoea, gastritis, colitis, hepatosplenomegaly, } \\
\text { lymphadenopathy, anaemia, thrombocytopenia, bedwetting, GLILD, APDS type } 1\end{array}$ \\
\hline 11 & $\mathrm{~F}$ & 13 & $\begin{array}{l}\text { Pneumonia, sinusitis, chronic bronchitis, otitis media, pharyngitis, bronchiectasis, bronchial asthma, } \\
\text { lymphadenopathy, growth failure }\end{array}$ \\
\hline 12 & $\mathrm{~F}$ & 13 & Pneumonia, sinusitis, pharyngitis, lymphadenitis, acne vulgaris, pemphigus, leukopenia \\
\hline 13 & $\mathrm{~F}$ & 16.5 & Pneumonia, sinusitis, otitis media, bronchial asthma \\
\hline 14 & M & 10 & Pneumonia, bronchitis, stomatitis \\
\hline
\end{tabular}

at different times from diagnosis, they were switched from intravenous to subcutaneous therapy with a good clinical effect. We also assessed their IgG levels and infection frequency. One patient had to return to intravenous therapy due to severe allergic reactions (urticaria on the skin) and general symptoms (fever, headaches) related to the transfusion of subcutaneous preparations.

A health condition of 13 out of 14 patients (92.85\%) improved (lower respiratory tract infections resolved or their frequency was significantly reduced) after the institution of human immunoglobulin replacement therapy regardless of the administration route. There was no improvement in symptoms of chronic sinusitis with intermittent exacerbations. In 2 (21.4\%) boys, severe bronchial asthma and symptoms of atopic dermatitis significantly improved. Autoimmune symptoms and lymphoproliferation features did not resolve, but their worsening was not observed either.

The replacement therapy did not improve the health condition of one boy (7.14\%). Despite the IgG level in the range from $7 \mathrm{~g} / \mathrm{l}$ to $10 \mathrm{~g} / \mathrm{l}$, problematic infections and massive lymphoproliferation (hepatosplenomegaly, lymphadenopathy, hyperplasia of lymphoid follicles in the gastric mucosa) were still present. For this reason, diagnostics were performed outside the country. NGS tests helped to identify a mutation that allowed for the diagnosis of a combined immunodeficiency (APDS type 1).
The boy underwent allogeneic hematopoietic cell transplantation from a matched family donor (his brother). Complete immunological reconstruction was achieved, with the possibility of completing the replacement therapy approximately 2 years after the transplantation. All clinical signs of immunodeficiency resolved, except for chronic sinusitis.

\section{Discussion}

Common variable immunodeficiency is a heterogeneous disease dominated by recurrent respiratory tract infections, non-malignant lymphoproliferation, autoimmune diseases, often in the form of thrombocytopenia, autoimmune haemolytic anaemia, and neoplastic diseases [2, 3, 6, 12, 17, 18, 21-23]. The results of the presented study confirm the diverse clinical course of CVID. Due to the unique sets of symptoms and comorbidities, the possibility of PID was considered relatively late. However, the delay in children was shorter than in adults, which is consistent with literature data $[22,24,25]$. The delay in final diagnosis in our centre was between 1.5 and 7 years in a child with suspected immunodeficiency, and between 5 and 18 years in an adult. The median for the entire group was 5 years. The diagnostic delay in the patients presented in the study by Mohammadinejad et al. [11] was 4.4 years on average (SD 3.59) and was significantly 
Table 4. The incidence of individual symptoms in patients with CVID

\begin{tabular}{|c|c|c|}
\hline Clinical symptoms & $\begin{array}{l}\text { Number of } \\
\text { patients with the } \\
\text { symptom }(n=14)\end{array}$ & $\begin{array}{c}\text { Percentage } \\
\text { (\%) }\end{array}$ \\
\hline Respiratory system: & 14 & 100 \\
\hline Pneumonia & 12 & 85.7 \\
\hline Bronchitis & 12 & 85.7 \\
\hline Pharyngitis & 10 & 71.4 \\
\hline Sinusitis & 9 & 64.2 \\
\hline Otitis media & 9 & 64.2 \\
\hline Bronchiectasis & 4 & 28.5 \\
\hline Asthma & 2 & 14.3 \\
\hline Tuberculosis & 1 & 7.1 \\
\hline Lung cancer & 1 & 7.1 \\
\hline Hematopoietic system: & 9 & 64.2 \\
\hline Lymphadenopathy & 6 & 42.8 \\
\hline Hepatomegaly & 4 & 28.5 \\
\hline Anaemia & 4 & 28.5 \\
\hline Leukopenia & 2 & 21.4 \\
\hline Thrombocytopenia & 2 & 21.4 \\
\hline Splenomegaly & 1 & 7.14 \\
\hline Digestive system: & 4 & 28.5 \\
\hline Diarrheal & 4 & 28.5 \\
\hline Esophagitis & 1 & 7.1 \\
\hline Colitis & 1 & 7.1 \\
\hline Crohn's disease & 1 & 7.1 \\
\hline Skin: & 5 & 35.7 \\
\hline Atopic dermatitis & 3 & 21.4 \\
\hline Acne vulgaris & 3 & 21.4 \\
\hline Hives & 3 & 21.4 \\
\hline Alopecia & 1 & 7.14 \\
\hline Cellulitis & 1 & 7.14 \\
\hline Urinary tract: & 4 & 28.5 \\
\hline Urinary tract infection & 4 & 28.5 \\
\hline Erythrocyturia & 2 & 14.3 \\
\hline Other: & 2 & 14.3 \\
\hline Candidiasis & 2 & 14.3 \\
\hline
\end{tabular}

shorter than in patients diagnosed several years earlier (5.1 years on average). In England, the delay in diagnosis of CVID was 2.5 years, and in the USA - 4 to 6 years [11]. Based on the clinical analysis of 2212 patients presented in the study by Gathmann et al., the diagnostic delay varied by country, however, for all patients combined, the median delay was 4.1 years. The shortest diagnostic delay was 1.8 years and was observed in children from
Table 5. Immunoglobulin values in main classes at diagnosis, after 3 months of intravenous transfusions and at the time of transition to subcutaneous immunoglobulins in individual patients

\begin{tabular}{|c|c|c|c|c|c|}
\hline \multirow[t]{2}{*}{ Patient } & \multicolumn{3}{|c|}{$\begin{array}{c}\text { Immunoglobulin } \\
\text { levels at diagnosis } \\
{[\mathrm{g} / \mathrm{l}]}\end{array}$} & \multirow{2}{*}{$\begin{array}{l}\text { IgG level after } \\
3 \text { months of } \\
\text { intravenous } \\
\text { transfusions } \\
{[\mathrm{g} / \mathrm{l}]}\end{array}$} & \multirow{2}{*}{$\begin{array}{c}\text { IgG level at } \\
\text { transition to the } \\
\text { subcutaneous route } \\
\text { of administration } \\
{[\mathrm{g} / \mathrm{l}]}\end{array}$} \\
\hline & IgG & $\operatorname{Ig} A$ & $\lg M$ & & \\
\hline 1 & 4 & 0.06 & 0.3 & 6.8 & 6.1 \\
\hline 2 & 0.4 & 0.06 & 0.4 & 8.3 & 6.8 \\
\hline 3 & 4.34 & 0.06 & 0.3 & 6.6 & 6 \\
\hline 4 & 4.8 & 0.5 & 1.3 & 6.4 & 5.2 \\
\hline 5 & 0.9 & 0.06 & 0.04 & * & * \\
\hline 6 & 0.2 & 0.06 & 0.17 & 7 & 7 \\
\hline 7 & 3.2 & 0.06 & 0.27 & 7.3 & 7.7 \\
\hline 8 & 0.1 & 0.5 & 1.3 & 6.6 & 5.2 \\
\hline 9 & 0.5 & 0.06 & 0.12 & 10 & 8.3 \\
\hline 10 & 1.56 & 0.33 & 2.3 & 9.8 & 6 \\
\hline 11 & 6 & 0.05 & 0.38 & 10.5 & 9.2 \\
\hline 12 & 1.42 & 0.05 & 0.18 & 10.3 & 8.3 \\
\hline 13 & 6.4 & 3.2 & 0.13 & 11 & 8.2 \\
\hline 14 & 1.8 & 0.2 & 0.8 & 6.8 & 5.8 \\
\hline Median & 1.68 & 0.06 & 0.3 & 7.3 & 6.8 \\
\hline Mean & 2.54 & 0.38 & 0.57 & 8.26 & 6.9 \\
\hline SD & 2.21 & 0.83 & 0.64 & 1.78 & 1.31 \\
\hline Min. & 0.1 & 0.05 & 0.04 & 1.78 & 5.2 \\
\hline Max. & 6.4 & 3.2 & 2.3 & 11.0 & 9.2 \\
\hline
\end{tabular}

*The patient was transferred to another medical centre.

Poland [9]. According to the analysis by Maarschalk et al., the longest delay was observed in patients with the dominant autoimmune phenotype and was 17 years [1].

The clinical phenotype demonstrated in our patients was similar to that in other analysed populations of patients with CVID. Recurrent upper and lower respiratory tract infections predominated. In $85.7 \%$ of patients, pneumonia was diagnosed at least once, including complicated pneumonia in 3 patients (respiratory failure, lung empyema, inflammatory tumour requiring surgical resection). Sinusitis was diagnosed in $64.2 \%$ of patients, including the necessity to perform functional endoscopic sinus surgery (FESS) in several patients. This is in line with the current literature. According to Jolles [23], recurrent bacterial respiratory tract infections occurred in $90 \%$ of patients with CVID, and the most common diagnoses were pneumonia and sinusitis (over $70 \%$ of patients). In Iranian patients, pneumonia (31.9\%) and sinusitis (18.8\%) 
Table 6. Lymphocyte subpopulation results in patients with CVID at diagnosis

\begin{tabular}{lcccc}
\hline Patient & CD3+ $(\% / \mu l)$ & CD3+CD4+ $\% / \mu l)$ & CD3+CD8+ $\% / \mu l)$ & CD 19+ $(\% / \mu l)$ \\
\hline 1 & $70.05 / 855$ & $51.02 / 623$ & $23 / 281$ & $8.39 / 101$ \\
\hline 2 & $78.1 / 1464$ & $52.30 / 981$ & $23.60 / 443$ & $10.7 / 201$ \\
\hline 4 & $77.21 / 3.62$ & $17.52 / 0.82$ & $57.57 / 2.7$ & $0.3 / 0.01$ \\
\hline 5 & $72.3 / 1196$ & $35.6 / 589$ & $29.3 / 485$ & $9 / 149$ \\
\hline 6 & $73.3 / 344$ & $32.8 / 154$ & $38.5 / 181$ & $5.6 / 26$ \\
\hline 7 & $74.9 / 2902$ & $21.5 / 833$ & $45.6 / 1767$ & $22.3 / 864$ \\
\hline 8 & $69.09 / 1350$ & $41.2 / 806$ & $20.19 / 393$ & $18.98 / 370$ \\
\hline 9 & $68.3 / 848$ & $35.1 / 436$ & $26.8 / 333$ & $19.90 / 350$ \\
\hline 10 & $84.1 / 6731$ & $31.4 / 2513$ & $50.6 / 4050$ & $12.4 / 992$ \\
\hline 11 & $78.9 / 1515$ & $15.8 / 303$ & $49.8 / 956$ & $5.9 / 113$ \\
\hline 12 & $53.17 / 994$ & $14.67 / 273$ & $36.3 / 680$ & $6.87 / 127$ \\
\hline 13 & $71.97 / 1326$ & $40.30 / 743$ & $29.76 / 548$ & $8.23 / 151$ \\
\hline 14 & $75.7 / 978$ & $36.1 / 466$ & $37.2 / 481$ & $16.2 / 209$ \\
\hline Min. & $72.5 / 2208$ & $25.5 / 777$ & $35.3 / 1075$ & $5.4 / 164$ \\
\hline Max. & $53.17 / 3.62$ & $14.7 / 0.82$ & $20.19 / 2.7$ & $0.3 / 0.01$ \\
\hline Mean & $84.1 / 6731$ & $52.3 / 2513$ & $57.57 / 4050$ & $22.3 / 992$ \\
\hline Median & $72.8 / 1622.47$ & $32.2 / 678$ & $35.9 / 834$ & $10.72 / 272.6$ \\
\hline SD & $72.9 / 1261$ & $33.95 / 606$ & $35.8 / 483$ & $8.7 / 157.5$ \\
\hline & $7.089 / 1634.063$ & $12.06 / 599$ & $6.4 / 296.8$ \\
\hline
\end{tabular}

were also most frequently diagnosed [11]. Similarly, Gathmann et al. showed pneumonia in $32 \%$ of patients. It is a significant study because it included a large number of patients with diagnosed CVID [9].

Both our own and literature data confirm that lung diseases are the most common problem in patients with CVID and lead to increased morbidity and mortality. Infections caused by the encapsulated bacteria such as Haemophilus influenzae or Streptococcus pneumoniae remain particularly dangerous [1]. We did not analyse the aetiology of infections in our patients (lack of data in retrospective analyses).

Both our own study and the literature data show that patients with CVID have non-infectious problems [1, 9, 12, 23]. In the presented patient group, autoimmunity features in various forms (alopecia, lupus-like syndrome, immune thrombocytopenia) occurred in $42.9 \%$ and noninfectious diseases of the gastrointestinal tract were observed in $14.3 \%$ of patients. In the study by Gathmann et al. [9], autoimmunity was shown in $29 \%$ of patients and splenomegaly in $26 \%$. The analysis of a large group of 2212 patients showed that splenomegaly, autoimmunity and enteropathy are closely related and are some of the most significant symptoms of CVID [9].

In the presented group of 14 patients with CVID, neoplastic disease was diagnosed in 1 adult, the oldest patient, in whom the delay in diagnosis was 18 years. Additionally, the patient was a long-time cigarette smoker.
This probably contributed to the development of lung cancer. According to the literature data, lymphomas and gastric cancer are much more frequently diagnosed in patients with CVID $[9,15]$.

At the moment of diagnosis, immunoglobulin levels in the main classes were very low in most of the presented patients. Only individual patients presented the CVID phenotype with a slight reduction in IgG, IgM and/or IgA levels, which is consistent with the literature data. There are also reports that extremely low immunoglobulin levels of all or some major classes are associated with a higher risk of complications, e.g. bronchiectasis was more common in patients with significantly lower immunoglobulin $M$ levels, pneumonia and meningitis were more often associated with low levels of immunoglobulin $G$. Enteropathy was demonstrated much more often in patients with the lack of immunoglobulin $M$ and $A$ [9, 15].

The most important thing in the treatment of patients with CVID is the adequate supply of human intravenous immunoglobulins. The recommended dose is $0.2-0.8 \mathrm{~g} / \mathrm{kg} \mathrm{bw} / \mathrm{month}$. All the presented patients started replacement therapy with intravenous preparations. During this time, IgG levels increased to the expected values (usually within the age range), allowing for good infection control. The substitution of immunoglobulins did not significantly affect the distribution of the basic lymphocyte subpopulations, which is consistent with the literature data $[1,11,26]$. 
After approximately 3 months of intravenous therapy, patients continued subcutaneous human immunoglobulins treatment. After proper training, a patient can carry out such therapy independently at home. Our own experience shows that subcutaneous and intravenous therapies are equally effective. On the other hand, subcutaneous administration is considered to be safer, less frequently causing side effects $[4,12,26]$. However, in the presented group of patients, one boy had to return to intravenous therapy due to the side effects after subcutaneous administration. The boy tolerated intravenous immunoglobulin supplementation well.

After the institution of human immunoglobulin replacement therapy, a significant clinical improvement was achieved in 13 out of 14 patients, consisting in a reduction in the frequency of infections, especially in the lower respiratory tract. Only one boy did not achieve the expected benefits of the treatment. In this patient, NGSbased genetic testing allowed to change the diagnosis from CVID to APDS type 1. It is a combined immunodeficiency, which explains why the immunoglobulin treatment was not sufficient in this child. Curing of immunodeficiency was possible thanks to the hematopoietic cell transplantation from a healthy, related donor (his brother). In all of the presented patients, the implemented immunoglobulin replacement therapy resulted in a reduction in the quantity and quality of respiratory or gastrointestinal infections.

An important information, resulting from our own observations, is the fact that the presence of dysmorphia, developmental disorders or the lack of the expected improvement after treatment are a strong indication for conducting NGS-based genetic testing. The similar phenotypes of diseases classified as PID make direct sequencing of single genes ineffective. On the other hand, still limited access and, above all, the lack of financing by the National Health Fund for genetic tests are the reasons why it is difficult to recommend NGS testing in all patients diagnosed with CVID. Among our patients, one boy diagnosed at 17 years of age had an extremely low percentage and absolute number of CD19+ cells. This raises a suspicion that he may be suffering from Bruton agammaglobulinemia. In this situation, Sanger sequencing of the Bruton tyrosine kinase gene would be reasonable to resolve the doubts. There are reports of XLA patients with a milder phenotype and a late diagnosis [25, 27].

\section{Conclusions}

Common variable immunodeficiency is a disease that occurs at any age. It is characterized by a heterogeneous clinical picture, but the infectious manifestation is most common in children and adults. The diagnostic delay is still too long, especially in adults, which can lead to serious and irreversible complications. Early diagnosis and appropriate treatment with intravenous and subcutane- ous immunoglobulins reduces the frequency of infections and their potential complications. Atypical symptoms of CVID, increased prevalence of autoimmune phenomena, non-malignant lymphoproliferation as well as the lack of the expected improvement after proper treatment are a strong indication for NGS-based genetic testing.

\section{Conflict of interest}

The authors declare no conflict of interest.

\section{References}

1. Maarschalk-Ellerbroek LJ, Hoepelman Al, van Montfrans JM, Ellerbroek PM. The spectrum of disease manifestations in patients with common variable immunodeficiency disorders and partial antibody deficiency in a university hospital. J Clin Immunol 2012; 32: 907-21.

2. Azizi G, Abolhassani H, Asgardoon MH, et al. Autoimmunity in common variable immunodeficiency: epidemiology, pathophysiology and management. Expert Rev Clin Immunol 2017; 13: 101-15.

3. Cunningham-Rudles C. Common variable immune deficiency: case studies. Hematology Am Soc Hematol Educ Program 2019; 2019: 449-56.

4. Abolhassani H, Sagvand BT, Shokuhfar T, et al. A review on guidelines for management and treatment of common variable immunodeficiency. Expert Rev Clin Immunol 2013; 9: 561-74.

5. Patuzzo G, Barbieri A, Tinazzi E, et al. Autoimmunity and infection in common variable immunodeficiency (CVID). Autoimmun Rev 2016; 15: 877-82.

6. Sekinaka Y, Mitsuiki N, Imai K, et al. Common variable immunodeficiency caused by FANC mutations. J Clin Immunol 2017; 37: 434-44.

7. Warnatz K, Bossaller L, Salzer U, et al. Human ICOS deficiency abrogates the germinal center reaction and provides a monogenic model for common variable immunodeficiency. Blood 2006; 107: 3045-52.

8. Li R, Zheng Y, Li Y, et al. Common variable immunodeficiency with genetic defects identified by whole exome sequencing. Biomed Res Int 2018; 2018: 3724630.

9. Gathmann B, Mahlaoui N, CEREDIH, Gérard L, et al. European Society for Immunodeficiencies Registry Working Party: clinical picture and treatment of 2212 patients with common variable immunodeficiency. J Allergy Clin Immunol 2014; 134: 116-26.

10. Azizi G, Abolhassani H, Mahdaviani SA, et al. Clinical, immunologic, molecular analyses and outcomes of Iranian patients with LRBA deficiency: a longitudinal study. Pediatr Allergy Immunol 2017; 28: 478-84.

11. Mohammadinejad P, Aghamohammadi A, Abolhassani H, et al. Pediatric patients with common variable immunodeficiency: long-term follow-up. J Investig Allergol Clin Immunol 2012; 22: 208-14.

12. Grześk E, Dąbrowska A, Urbańczyk A, et al. Common variable immunodeficiency disorders - diseases with many faces. Pediatr Pol 2018; 93: 186-91.

13. Cunningham-Rundles $C$. The many faces of common variable immunodeficiency. Hematology Am Soc Hematol Educ Program 2012; 2012: 301-5.

14. Salzer U, Warnatz K, Peter HH. Common variable immunodeficiency: an update. Arthritis Res Ther 2012; 14: 223. 
15. Varricchi G, Pecoraro A, Crescenzi L, et al. Gastroduodenal disorders in patients with CVID undergoing immunoglobulin therapy. Curr Pharm Biotechnol 2018; 19: 734-41.

16. Conaway E. Novel variant of common variable immunodeficiency. BMJ Case Rep 2017; 2017. pii: bcr-2017-219655. doi: 10.1136/bcr-2017-219655.

17. Azizi G, Tavakol M, Rafiemanesh $\mathrm{H}$, et al. Autoimmunity in a cohort of 471 patients with primary antibody deficiencies. Expert Rev Clin Immunol 2017; 13: 1099-106.

18. Tak Manesh A, Azizi G, Heydari A, et al. Epidemiology and pathophysiology of malignancy in common variable immunodeficiency? Allergol Immunopathol 2017; 45: 602-15.

19. Ameratunga R, Brewerton M, Slade C, et al. Comparison of diagnostic criteria for common variable immunodeficiency disorder. Front Immunol 2014; 5: 415.

20. http://esid.org/Working-Parties/Registry/Diagnosis-criteria 25.04.2017.

21. Gobert D, Bussel JB, Cunningham-Rundles C, et al. Efficacy and safety of rituximab in common variable immunodeficiency-associated immune cytopenias: a retrospective multicentre study on 33 patients. Br J Haematol 2011; 155: 498-508.

22. Rae W. Indications to epigenetic dysfunction in the pathogenesis of common variable immunodeficiency. Arch Immunol Ther Exp 2017; 65: 101-10.

23. Jolles $\mathrm{S}$. The variable in common variable immunodeficiency: a disease of complex phenotypes. J Allergy Clin Immunol Pract 2013; 1: 545-56.

24. Lankisch P, Schiffner J, Ghosh S, et al. The Duesseldorf warning signs for primary immunodeficiency: is it time to change the rules? J Clin Immunol 2015; 35: 273-9.

25. Gülsüm A, Sevgi K, Ismail R. Evaluation of clinical and immunological characteristics of children with common variable immunodeficiency. Int J Pediatr 2018; 2018: 3527480.

26. Pac M, Bernatowska E. Comprehensive activities to increase recognition of primary immunodeficiency and access to immunoglobulin replacement therapy in Poland. Eur J Pediatr 2016; 175: 1099-105.

27. Sigmon JR, Kasasbeh E, Krishnaswamy G. X-linked agammaglobulinemia diagnosed late in life: case report and review of the literature. Clin Mol Allergy 2008; 6: 5. 